\title{
El podcast como herramienta de enseñanza en física y química de bachillerato
}

\author{
Felipe QuinTANAL PÉREZ \\ Colegio Marista "La Inmaculada". (Granada) \\ fqyfqyfq@gmail.com
}

Recibido: $19 / 07 / 2012$

Aceptado: 31/10/2012

\begin{abstract}
Resumen
El presente artículo muestra la aplicación de los podcast a la enseñanza de la Física y Química de Bachillerato. Para ello se presenta la planificación seguida con los podcast, su secuenciación a lo largo del curso académico 2011 - 2012, la aplicación tecnológica empleada (Audacity), otros recursos temáticos utilizados en este proyecto como la elaboración de líneas del tiempo, vídeos sobre recetas de cocina y murales multimedia con los soportes informáticos usados, Dipity, Video Maker y Glogster, las rúbricas de evaluación seguidas, los resultados obtenidos, que han sido muy satisfactorios y las conclusiones emitidas.

Palabras clave: Podcast, Herramientas Web 2.0, Enseñanza, Física y Química.
\end{abstract}

\section{Podcast as a tool of teaching physics and chemistry of high school}

\begin{abstract}
This article shows the application of podcast to the teaching of physics and chemistry of high school. This planning is followed with the podcast, its sequencing throughout the academic year 2011-2012, (Audacity) employed technological application, other thematic resources employed in this project such as the development of timelines, videos on cuisine and recipes murals multimedia computer storage media used, Dipity, Video Maker with Glogster, the row headings of evaluation, the results have been very satisfactory and issued conclusions.
\end{abstract}

Keywords: Podcast, Web 2.0 tools, Teaching, Physics and Chemistry.

\section{Referencia normalizada}

QUINTANAL PEREZ Felipe (2012): "El podcast como herramienta de enseñanza en física y química de bachillerato”. Estudios sobre el mensaje periodístico. Vol. 18, núm. especial noviembre, págs.: 729738. Madrid, Servicio de Publicaciones de la Universidad Complutense.

Sumario: 1. Introducción; 1.1 Origen de la investigación; 1.2 Fundamentación y contexto de la investigación; 1.3 Podcast educativos y orientaciones didácticas para su realización. 2. Metodología empleada. 3. Desarrollo efectuado; 3.1 Etapa inicial; 3.2 Etapa intermedia; 3.3 Etapa final. 4. Resultados obtenidos; 4.1 Productos elaborados por los alumnos; 4.2 Valoraciones realizadas por los estudiantes; 4.3 Valoración efectuada por el profesor. 5. Conclusiones. 6. Referencias bibliográficas

\section{Introducción}

\subsection{Origen de la investigación}

El origen de esta investigación se ancla en dos raíces poderosas. La primera recoge las lecturas previas efectuadas que han suscitado una serie de nuevas preguntas y nuestro interés por generar nuevas ideas. La segunda ha sido nuestra propia experiencia, que desde el conocimiento del contexto en el que nos movemos y de los hechos educativos que acaecen, nos ha permitido sugerir respuestas a los problemas detectados en la praxis.

Secundariamente, la investigación presenta también un fundamento metodológico, pues desde hace años, las asignaturas relacionadas con la ciencia escolar son cuestio- 
nadas por la didáctica empleada por el profesorado, el interés que suscita en los alumnos y por el rendimiento académico obtenido.

En último lugar, aparece una razón emotiva como es nuestro cariño personal hacia la Ciencia, lo que nos dirige hacia el compromiso en la búsqueda de medios y de acciones educativas que puedan aportar soluciones en los nuevos escenarios educativos.

\subsection{Fundamentación y contexto de la investigación}

La fundamentación de nuestra investigación se apoya en un hecho irrefutable al cual se enfrenta la enseñanza-aprendizaje de la ciencia en la escuela y la didáctica que emana de esta ciencia escolar. Dicho hecho es la depreciación actitudinal por parte del alumnado hacia las asignaturas científicas que, desde hace algunos años, se traduce en una menor elección orientada hacia carreras científicas.

Además, esta depreciación se ve acompañada de un perfil temporal variable cuyo mínimo acontece en la adolescencia según apuntan diferentes investigaciones y estudios como sugiere (Quintanal, 2011: 154). Ello conduce hacia una menor opción por asignaturas de corte científico "duro" y, subsiguientemente, a un decaimiento del número de vocaciones científicas, sobre todo, de género femenino.

Para ahondar más en esta crisis de vocaciones científicas se une a la visión que se ofrece de la Ciencia en muchos medios de comunicación como foco y fuente de problemas medioambientales complejos, la propia psicología del adolescente, lo que cierra el ciclo del diagnóstico negativo actitudinal de los estudiantes.

Por otra parte, el propio sistema educativo no ayuda a la mejora de esta percepción. Pedagogías tradicionales expositivas, escasa relación entre los hechos científicos y la vida cotidiana del alumno, currícula excesivamente densos, uso insuficiente de las TIC, ausencia de trabajo en el laboratorio, número de horas lectivas bajo, excesiva orientación en clase para aprobar exámenes, tipología del profesorado constituyen algunos factores influyentes que ratifican al estudiante en su percepción de la Ciencia como algo difícil, autoritario y aburrido.

Con objeto de minimizar el influjo de estos agentes se ha situado el problema descrito en nuestra investigación dentro del contexto de un centro privado-concertado, en el que desempeña su labor el investigador y la etapa escogida para ser consolidada, Bachillerato. El curso seleccionado ha sido $1^{\circ}$ y los alumnos, los pertenecientes a las modalidades de Salud y Tecnología.

\subsection{Podcast educativos y orientaciones didácticas para su realización}

El podcast, según (Wikipedia, 2012) "se asemeja a una suscripción a un blog hablado en la que recibimos los programas a través de Internet. Su contenido es tan diverso como la radio tradicional incluyendo noticias, documentales, música, debates, entrevistas, etc. Mucha gente prefiere usar un guión y otros hablan de forma improvisada. Algunos parecen un programa de radio, intercalando música, mientras que otros hacen podcast más cortos y exclusivamente con voz, igual que con los videoblogs".

Los podcast, como herramientas de comunicación, suelen utilizar los géneros periodísticos, definidos de diversas maneras por los expertos del tema. Si nos atenemos a la exposición oral del locutor, se pueden clasificar en dos géneros: monólogo y diálogo. 
El género monólogo abarca "la noticia, el informe, la crónica, el editorial y la información" (Merayo, 1992: 167) mientras que el género diálogo comprende "la noticia dialogada, el reportaje, la crónica de alcance, la entrevista, el coloquio y la participación" (Merayo, 1992: 186).

Pero una cosa es la teoría y otra la praxis por lo que se debe tener en cuenta que muchos podcasters se dejan llevar por su libertad creativa o por el desconocimiento de los géneros informativos $y$, por ende, ciertas estructuras periodísticas son omitidas o se dan mezcladas.

Debido a los diferentes recursos aportados en Internet, la tipología de los podcast es muy versátil. Una posible forma de clasificarlos puede ser la siguiente:

- Podcast informativo. Se dedican a la sencilla exposición de datos y a la extracción de declaraciones de las entrevistas realizadas. Su interacción con el oyente no es tan elevada como en otros tipos de podcast.

- Podcast interpretativo. No se suelen hacer crónicas o reportajes en los podcast, pero eso no quiere decir que no existan en ellos. Une todo tipo de géneros.

- Podcast de opinión. Hace referencia al tipo de contenido, algo personal, que se trata en el programa. Hay quien lo llama "audiopost" y precisa de una elaboración compleja.

Para que el podcast se considere educativo debe tratarse de un elemento pedagógico que implique la existencia de un archivo sonoro con contenidos educativos, elaborado tras una planificación previa. Puede ser editado por un docente, un alumno, una empresa o una institución educativa.

Las grandes ventajas de los podcast educativos son, según (Solano y Sánchez, 2010: 128):

- La difusión de contenidos de audio de forma sencilla mediante una arquitectura web.

- La repetición y revisión ilimitada de los procesos de escucha y visionado, en el caso de los videocast.

- La promoción del intercambio del conocimiento y su adaptación a diferentes contextos educativos mediante el desarrollo de contenidos abiertos.

- La posibilidad de dirigirse a un público especializado o genérico.

- La distribución periódica de contenidos empleando los sistemas de sindicación existentes.

- La entrada de información sobre las modificaciones efectuadas por docentes, alumnos o profesionales.

- La posibilidad de diversificar los recursos de enseñanza empleados.

Por todo ello, el podcast se puede convertir en una herramienta muy potente y flexible para la educación porque nos permite elaborar guiones adaptados a nuestros ámbitos educativos. Se hace necesario, por tanto, tener presente algunas orientaciones didácticas a la hora de efectuar los podcast para no disipar su potencialidad. 
Dichas orientaciones abarcan los ámbitos tecnológico y didáctico. El ámbito tecnológico se refiere a las técnicas y aplicaciones que se pueden usar. La realización del podcast desde este punto de vista se puede secuenciar en cinco fases que se muestran en la Figura 1.

Figura 1: Etapas para la elaboración tecnológica de un podcast educativo. Fuente: Prodinpa-Feditic, 2010

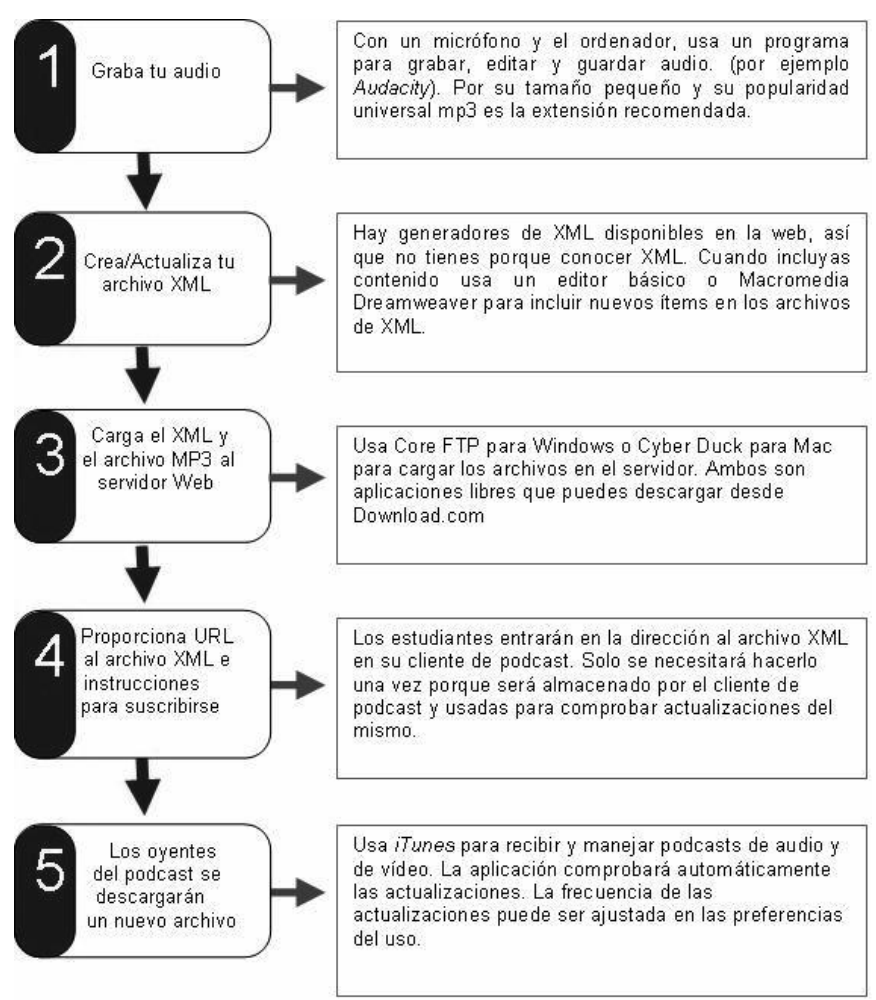

A nivel pedagógico, la producción de un podcast educativo debe responder a una fase previa de reflexión y otra de secuenciación de actividades. La etapa de reflexión debe recoger la previsión efectuada sobre la adecuación de la herramienta que vamos a usar en la clase, así como las características, motivaciones, competencias e intereses de los alumnos. La secuenciación didáctica debe recoger la descripción de la actividad, las tareas, fases y temporalización, la metodología empleada, los recursos a utilizar y la evaluación (criterios e instrumento de evaluación).

La tipología de actividades que se puede desarrollar es amplia y variable. Destacan, según (Solano y Amat, 2008: 8), las siguientes: libro auditivo, entrevistas formales o informales, grabaciones de exposiciones teóricas, de clases magistrales, taller de periodismo escolar, taller de radio escolar, ponencias o conferencias grabadas, grabación de exámenes, revista académica, tutoriales, podcast para formación de maestros y recursos web para formación de formadores.

\section{Metodología empleada}

El proyecto se desarrolló durante el curso 2011-2012 y estuvo dirigido a los alumnos de las modalidades científico-tecnológica de $1^{\circ}$ de Bachillerato. El número de alumnos implicados fue de 50 alumnos, 19 alumnas y 31 alumnos. Del total, 26 pertenecían a la modalidad primera, compuesta por 14 alumnas y 12 alumnos y 24 a la segunda, constituida por 5 chicas y 19 chicos. 
Tras el preceptivo diálogo con los alumnos, se escogió inicialmente la biografía de insignes científicos como temática para el proyecto y como herramienta Web 2.0 para desarrollarlo, Audacity (elaboración de podcast). Además, se elaboraron en castellano y en inglés, para apoyar la enseñanza del idioma.

Como plataforma de gestión de las tareas se usó Edmodo, que permitió establecer asignaciones, el envío de las tareas, su calificación, la realización de encuestas, la compartición de todos los trabajos y la comunicación profesor-alumno y entre alumnos.

Como el proyecto duró todo el curso, se fragmentó en tres etapas, introduciendo en las etapas segunda y tercera otras tareas diferentes a los podcast con objeto de evitar el tedio y la rutina que se podía generar. Así, se introdujeron la elaboración de líneas del tiempo, vídeos sobre recetas de cocina sencillas y murales multimedia, utilizando las siguientes herramientas Web 2.0, Dipity, Video Maker o similar y Glogster, respectivamente.

Se complementó el proyecto con la evaluación de los podcast y de los vídeos de recetas por parte del propio alumnado, así como el cumplimiento del cuestionario de autoevaluación.

\section{Desarrollo efectuado}

El proyecto se dividió en tres etapas o fases. La secuenciación de actividades y su cronología se explican a continuación.

\subsection{Etapa inicial}

Se cumplimentaron las siguientes tareas:

- Constitución de los equipos de trabajo (parejas).

- Selección de un científico importante y de una unidad didáctica del temario.

- Tarea 1: “¿Quién es quien?” Elaboración de un guión radiofónico sobre la biografía del científico seleccionado. Grabación y edición de dicho guión en formato podcast mediante el uso de Audacity.

- Tarea 2: "Física y Química en el bolsillo". Elaboración de un guión radiofónico sobre la unidad didáctica escogida. Grabación y edición de dicho guión en formato podcast mediante el uso de Audacity.

- Entrega de las tareas en la plataforma Edmodo.

- Comentario general del trabajo realizado a las clases. Comentario particular a algunos equipos a través de la red social.

\subsection{Etapa intermedia}

- Tarea 3: "Who is who?" Elaboración, en inglés, de un guión radiofónico sobre la biografía del científico seleccionado en la etapa anterior. Grabación y edición de dicho guión en formato podcast mediante el uso de Audacity.

- Tarea 4: "Tell me a Science story”. Elaboración, en inglés, de un guión radiofónico sobre curiosidades, anécdotas o leyendas del científico escogido. Grabación y edición de dicho guión en formato podcast mediante el uso de Audacity. 
- Tarea 5: "Línea del tiempo". Diseño y realización de un eje cronológico o línea del tiempo sobre los aspectos y fechas más importantes asociadas al científico seleccionado mediante la aplicación Dipity.

- Evaluación de los podcast de un equipo de trabajo por parte de otro equipo diferente.

- Entrega de las tareas en la plataforma Edmodo.

- Comentario general del trabajo realizado a las clases. Comentario particular a algunos equipos a través de la red social.

\subsection{Etapa final}

- Tarea 6: "Cocina con - Ciencia (I)”. Realización y grabación en vídeo de una receta fácil de cocina. Elaboración de un guión describiendo la receta y de otro guión que explique los procesos químicos y físicos desarrollados en la ejecución de la receta.

- Tarea 7: "Cocina con - Ciencia (II)". Grabación y edición de ambos guiones en formato podcast mediante el uso de Audacity.

- Tarea 8: "Presentando y evaluando". Creación de un mural multimedia con Glogster que sirva de presentación al trabajo efectuado durante el curso y evaluación de las tareas 6 y 7 de un equipo de trabajo por parte de otro equipo diferente.

- Tarea 9: "Autoevaluando". Realización individual del cuestionario de autoevaluación propuesto.

- Entrega de las tareas en la plataforma Edmodo.

- Comentario general del trabajo realizado a las clases. Comentario particular a algunos equipos a través de la red social.

Señalar que el cuestionario de autoevaluación desarrollado constaba de trece ítems, de opción múltiple y única (del uno al cinco, equivalentes a mala, regular, buena, muy buena o excelente). Se recogieron 49 cuestionarios y sólo un alumno no lo cumplimentó.

Con respecto a la temática del cuestionario, cinco ítems valoraban la experiencia de realizar las tareas encomendadas, cuatro evaluaban las herramientas usadas, tres, el tipo de tarea y uno, estimaba globalmente el trabajo del estudiante a lo largo del curso.

\section{Resultados obtenidos}

\subsection{Productos generados por los alumnos}

Se produjeron en total cinco podcast, tres en lengua castellana y dos en lengua inglesa, sus correspondientes guiones, una línea del tiempo, un vídeo y un mural multimedia por cada equipo de trabajo y a lo largo del curso.

Todas estas tareas fueron alojadas en Edmodo y pudieron ser visualizadas y evaluadas por los propios alumnos. Se pretendió dar mayor difusión a los podcast, proponiendo su sindicación, pero al solicitar consentimiento por parte de las familias, se negaron masivamente, alegando razones de privacidad y de minoría de edad; de ahí que éstos sólo estuvieron disponibles en la plataforma para los estudiantes y el profesor. 
Figura 2: Fragmento del guión para el podcast efectuado sobre Stephen Hawking. Fuente: Elaboración propia

\subsection{Valoraciones reali- zadas por los estudian- tes}

Se obtuvieron tras el cumplimiento del cuestionario elaborado para ello. Así, el bloque de ítems relacionado con la valoración de efectuar las diversas tareas fue considerado muy positivamente, aunque se produjeron leves diferencias.

\begin{tabular}{|c|c|c|c|}
\hline BLOQUE & TEXTO & DURACIÓN & AUDIO \\
\hline 1 & $\begin{array}{l}\text { [Voz de Stephen Hawking: } 30 "] \\
\text { - Alex: este sonido metálico } \\
\text { informatizado y casi robótico es la } \\
\text { voz de la mente y las ideas de } \\
\text { uno de los cientificos más } \\
\text { famosos de la historia. } \\
\text { Matemático, físico y astrónomo } \\
\text { Stephen Hawking es una de las } \\
\text { imágenes más impactantes que } \\
\text { tiene hoy en día la ciencia... } \\
\text { [Cuña musical corta:5"] } \\
\\
\text { - Gonzalo: pero empecemos por } \\
\text { el principio... y para ello hemos } \\
\text { de viajar al } 8 \text { de enero de } 1942 \text { (o } \\
\text { como le gusta decir a Hawking: } \\
\text { 300 años después de la muerte } \\
\text { de Galileo) pues este } 8 \text { de Enero } \\
\text { de un invierno frío y con la } \\
\text { Segunda Guerra Mundial en } \\
\text { pleno desarrollo, nace Stephen } \\
\text { Hawking en el seno de una } \\
\text { familia, recién mudada a Oxford. } \\
\text { Era una familia de clase media } \\
\text { con ambos padres médicos y un } \\
\text { abuelo que era un no muy buen } \\
\text { granjero. La familia pronto } \\
\text { regresó a Highgate, al norte de } \\
\text { Londres, donde Stephen inició }\end{array}$ & $\begin{array}{c}02: 45 \\
\text { Aproxim. }\end{array}$ & $\begin{array}{l}\text { Al terminar la } \\
\text { grabación de voz } \\
\text { de Stephen y la } \\
\text { introducción que } \\
\text { hace Alex empieza } \\
\text { a sonar La } \\
\text { Búsqueda de lanna } \\
\left(5^{\prime \prime}\right) \text { y se baja el } \\
\text { volumen para la } \\
\text { intervención de } \\
\text { Gonzalo. Acto } \\
\text { seguido interviene } \\
\text { Alex y al acabar se } \\
\text { vuelve a subir el } \\
\text { volumen de la } \\
\text { canción }\left(20^{\prime \prime}\right)\end{array}$ \\
\hline
\end{tabular}

Figura 3: Medias por sexo obtenidas para las diversas tareas. Fuente: Elaboración propia

MEDIAS POR SEXO DE LAS TAREAS EFECTUADAS

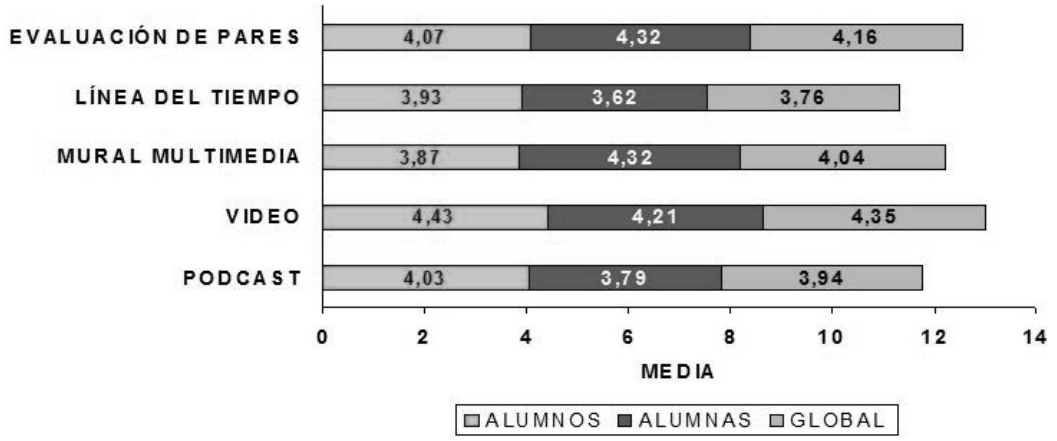

Se puede observar que las tareas de realización del vídeo, la evaluación de pares y el mural multimedia son las más valoradas en la muestra constituida por el grupo total, aunque por sexo se produzcan ligeras diferencias.

Así, los chicos valoran más la producción del vídeo y del podcast que las chicas, pero éstas valoran más positivamente el mural multimedia y la evaluación por pares que los chicos.

Ambos sexos coinciden en que la actividad menos valorada es la creación de la línea del tiempo. Al ser preguntados verbalmente en clase sobre esta cuestión, afir- 
maron que tuvieron problemas con la herramienta, ya que en el período de tiempo en el que la usaron, ésta se bloqueaba y no permitía hacer ni guardar cambios.

Con respecto a los ítems que valoraban las herramientas utilizadas, la Figura 4 recoge los resultados obtenidos.

Figura 4: Medias por sexo obtenidas para las diversas herramientas tecnológicas.

Fuente: Elaboración propia

MEDIAS POR SEXO DE LA VALORACIÓN DE LAS

HERRAMIENTAS TECNOLÓGICAS

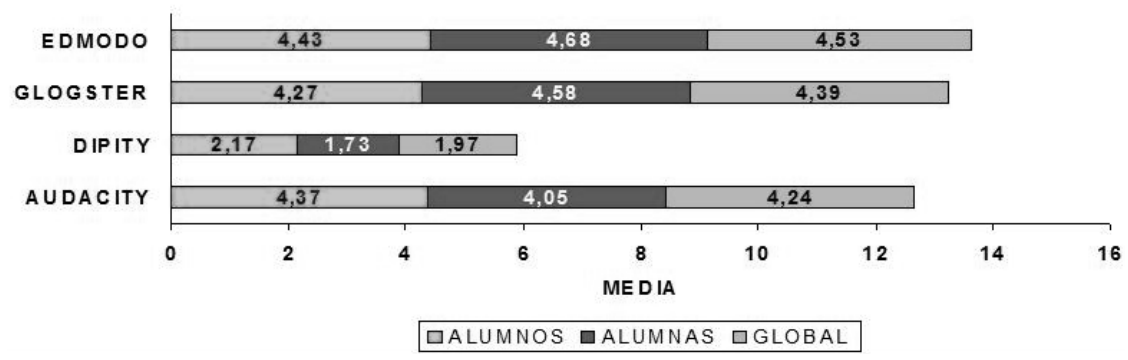

Se observa que globalmente la plataforma Edmodo es la mejor evaluada, seguida por Glogster y Audacity.

Por sexo, los alumnos valoran más Audacity que Glogster, invirtiéndose la tendencia en las alumnas. Creemos que hay una cierta coherencia en estos resultados, pues los chicos valoraban más positivamente la realización de podcast que las alumnas.

La herramienta Dipity es la peor valorada tanto por alumnos como por alumnas. Creemos que la mala experiencia que tuvieron con ella causada por el bloqueo y la lentitud de respuesta son los agentes originarios de esta evaluación.

Se debe comentar que no se ha evaluado la herramienta para realizar los vídeos, pues se dejó al libre albedrío de los equipos de trabajo. Por indagaciones posteriores sabemos que se utilizaron mayoritariamente Video Maker y Pinnacle Studio.

Por último, en la Figura 5 se muestran los resultados obtenidos sobre la valoración de las temáticas empleadas.

Figura 5: Medias por sexo obtenidas para las diversas temáticas. Fuente: Elaboración propia

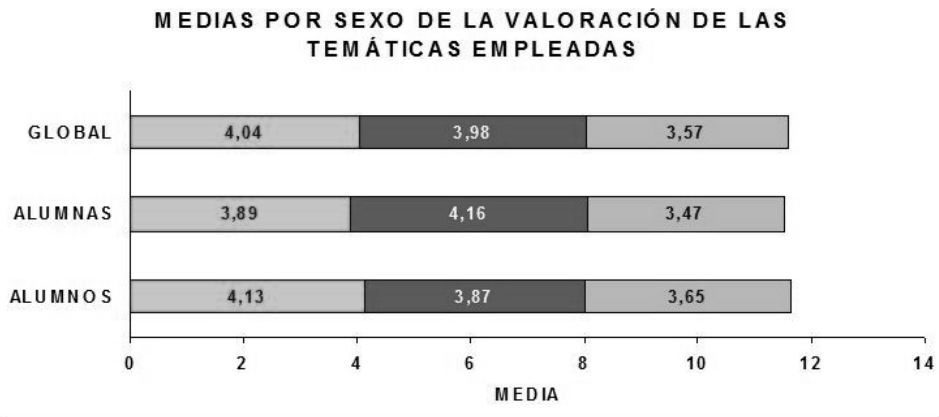

口BIOGRAFIAS CIENTIFIC OS םRECETAS COCINA Y PPOS. CIENTÍFICOS DLÍNEAS DEL TIEMPO 
Puede observarse que, en conjunto, la temática más valorada es el acercamiento a la vida de los científicos. Sin embargo, para las alumnas estuvo más adecuada la temática basada en la realización de una receta de cocina sencilla y sus principios científicos.

La construcción de líneas del tiempo es la temática menos apreciada por los estudiantes. En un diálogo posterior argumentaron que, después de la realización de podcast sobre científicos, los cronogramas no aportaban nada nuevo. Muchos de los alumnos también insistieron en que podía ser una temática más adecuada para otras materias, como por ejemplo, Historia.

En último lugar, se reseña que la percepción global sobre sus trabajos es muy alta, resultando una media de 4,22. Por sexo, los alumnos valoran más sus trabajos que las alumnas, calificándose con una media de 4,33 , mientras que las alumnas obtenían una media de 4,05 .

\subsection{Valoración efectuada por el profesor}

En general, muy satisfactoria. A tenor de lo que han comentado los estudiantes y de lo observado por el profesor hay coincidencia en la valoración global de la experiencia. No obstante, se han detectado ciertos bloqueos y ventajas que son interesantes explicar.

Los podcast iniciales resultaron tediosos y aburridos. Además, la gran mayoría de ellos usaron música comercial. En posterior diálogo con los alumnos, estos reconocieron que, apenas, habían visionado o leído los tutoriales sugeridos ni habían visitado las web aportadas para la descarga de música con licencia Creative Commons.

Estos inconvenientes fueron eludidos en la etapa intermedia. Para ello se proporcionó una rúbrica de evaluación a los estudiantes, clarificando qué había que hacer y cómo se iba a evaluar. Con esta medida se modificaron las deficiencias detectadas en la etapa inicial.

Otras limitaciones que detectamos fueron la imposibilidad de publicar los podcast y la exigua comunicación entre alumnos a través de Edmodo. Cuestionados posteriormente, algunos estudiantes adujeron que usaban otras redes (Tuenti) para comunicarse porque de esa manera tenían privacidad con respecto al profesor.

Entre las ventajas hay que señalar, que durante la etapa intermedia, se detectó un uso muy adecuado del software Audacity y de la plataforma Edmodo. La colaboración con el profesor de inglés se efectuó en este período, evaluando los guiones y los podcast que se hicieron en el idioma señalado.

En la etapa final se efectuó una colaboración con el profesor de lengua, al evaluar los guiones y los vídeos sobre recetas de cocina.

Se valoró también de forma positiva el hecho de haber efectuado otras tareas diferentes a los podcast. Se soslayó la saturación de los alumnos, permitiendo la utilización de otras herramientas Web 2.0, por lo que aumentaron sus habilidades tecnológicas y se reforzó el desarrollo de las habilidades comunicativas de los estudiantes, así como su motivación. 


\section{Conclusiones}

- Hemos considerado un podcast como un archivo sonoro que se puede escuchar en la red y /o descargado a nuestro ordenador o dispositivo portátil adecuado y al podcasting como al proceso de generar dicho archivo sonoro.

- El podcast presenta, por tanto, la ventaja de la ubicuidad, pues se puede escuchar en el momento que la persona desee, aunque los alumnos de la muestra explicitaron que no los escuchaban.

- Para aprovechar la potencialidad del podcast hay que efectuar un proceso previo que contemple, principalmente, los aspectos tecnológicos y didácticos.

- Coincidimos con (Fernández, 2007: 112) en que a la hora de emplear un medio con fines pedagógicos, se debe tener en cuenta los objetivos de aprendizaje planteados, las posibilidades del medio para conseguir dichos objetivos y la disponibilidad tecnológica de los estudiantes.

- Consideramos que el uso de podcast constituye una herramienta adecuada que, en la asignatura de Física-Química, permite mejorar las destrezas de expresión (oral, escrita) y de la comunicación.

\section{Referencias bibliográficas}

FERNÁNDEZ MORANTE, Carmen (2007): "El diseño y la producción de medios aplicados a la enseñanza", en CABERO ALMENARA, Julio (Coord.): Tecnología Educativa. Madrid, McGraw-Hill, pp. 102-122.

MERAYO PÉREZ, Arturo (1992): Para entender la radio. Salamanca, Servicio de Publicaciones de la Universidad Pontificia de Comillas, pp. 167-186.

PRODINPA - FEDITIC (2010): Wiki de la Universidad Nacional Francisco de Miranda, Falcón, Venezuela, en: http://prodinpafeditic.wikispaces.com/file/view /pasosParagrabarPodcast.JPG/147089609/pasosParagrabarPodcast.JPG. [fecha de consulta: 9 de septiembre de 2012].

QUINTANAL PÉREZ, Felipe (2011): "Estilos de aprendizaje y rendimiento académico en la Física y Química de Secundaria", en PEÑA ACUÑA, Beatriz (Coord.): Nuevas fórmulas docentes en el EEES. Madrid, Vision Libros, pp. 153-172.

SOLANO FERNÁNDEZ, Isabel Mª \& AMAT MUÑOZ, Laura M (2008): "Integración de podcast en contextos de enseñanza: criterios para el diseño de actividades", en DIGITUM, Biblioteca Universitaria: http://digitum.um.es/xmlui/handle /10201/14609. [fecha de consulta: 9 de septiembre de 2012].

SOLANO FERNÁNDEZ, Isabel $\mathrm{M}^{\mathrm{a}}$ \& SÁNCHEZ VERA, M${ }^{\mathrm{a}}$ del Mar (2010): "Aprendiendo en cualquier lugar: el podcast educativo", en Pixel-Bit, no 36: http://www.sav.us.es/pixelbit/pixelbit/articulos/n36/10.pdf. [fecha de consulta: 9 de septiembre de 2012].

WIKIPEDIA (2012): "Podcasting": http://es.wikipedia.org/wiki/Podcasting. [fecha de consulta: 9 de septiembre de 2012]. 


\section{Felipe QUINTANAL PÉREZ}

Colegio Marista "La Inmaculada”. Granada (España).

fqyfqyfq@gmail.com

Profesor de Física y Química de Secundaria y Bachillerato.

Doctor en Ciencias de la Educación por la UNED, Licenciado en Ciencias Químicas por la Universidad Hispalense de Sevilla y Master en Informática Educativa por la UNED. Su labor profesional se desarrolla como profesor de Ciencias en Secundaria y Bachillerato. Como investigador trabaja actualmente en la mejora de los estilos de aprendizaje aplicados a la Física y Química de Bachillerato y de la ESO empleando diversas herramientas Web 2.0 y en Programas de Innovación Docente de la Universidad de Granada, colaborando con el Departamento de Química Analítica de la Facultad de Ciencias. 